\title{
Circuit
}

Musiques contemporaines

\section{Notes pour Cantique de durées (Tremblay)}

\section{Gilles Tremblay}

Volume 6, numéro 1, 1995

Tremblay/Varèse/Messiaen : Gilles Tremblay analyste

URI : https://id.erudit.org/iderudit/902120ar

DOI : https://doi.org/10.7202/902120ar

Aller au sommaire du numéro

Éditeur(s)

Les Presses de l'Université de Montréal

ISSN

1183-1693 (imprimé)

1488-9692 (numérique)

Découvrir la revue

Citer cet article

Tremblay, G. (1995). Notes pour Cantique de durées (Tremblay). Circuit, 6(1), 43-50. https://doi.org/10.7202/902120ar

Ce document est protégé par la loi sur le droit d'auteur. L'utilisation des services d'Érudit (y compris la reproduction) est assujettie à sa politique d'utilisation que vous pouvez consulter en ligne.

https://apropos.erudit.org/fr/usagers/politique-dutilisation/
Cet article est diffusé et préservé par Érudit.

Érudit est un consortium interuniversitaire sans but lucratif composé de l'Université de Montréal, l'Université Laval et l'Université du Québec à Montréal. Il a pour mission la promotion et la valorisation de la recherche. https://www.erudit.org/fr/ 


\section{Notes pour Cantique de durées (Tremblay)}

Paru dans Revue d'esthétique, numéro spécial consacré aux musiques nouvelles, Paris, 1968, pp. 51-58.

Lorsqu'on regarde le ciel étoilé, les poudres palpitantes cernées de silence, l'œil, la pensée peuvent composer des ensembles imaginaires, grouper les phares sidéraux en de multiples figures. Aux constellations connues se superposent les contrepoints imaginés dans l'instant-contemplation. Prolonger en musique cet instant-contemplation, non pas le traduire - peut-on traduire l'indicible ? - tel est le rêve d'origine du Cantique de durées, de l'aventure que fut cette composition.

Contrairement à ce qui se passe pour les œuvres conçues par enchaînements dans le temps (une idée après l'autre), la composition fut élaborée par couches successives, le travail temporel étant fait en premier et conditionnant la matière même de la musique.

Au départ, sept durées (en doubles croches):

$\begin{array}{ccccccc}1 & 2 & 3 & 4 & 5 & 6 & 7 \\ 729 & 486 & 324 & 216 & 144 & 96 & 64\end{array}$

soit une progression fondée sur la relation 3-2 d'un intervalle de quinte fécond par excellence, puisqu'il épuise tous les sons du système tempéré avant de revenir au point de départ.

Ces sept durées donnent naissance par permutation à un réseau de sept formes-mères :

$\begin{array}{llllllll}\text { A) } & 1 & 2 & 3 & 4 & 5 & 6 & 7 \\ \text { B) } & 7 & 6 & 5 & 4 & 3 & 2 & 1 \\ \text { C) } & 4 & 7 & 1 & 6 & 2 & 5 & 3 \\ \text { D) } & 5 & 3 & 6 & 2 & 5 & 1 & 4 \\ \text { E) } & 6 & 1 & 7 & 5 & 3 & 4 & 2 \\ \text { F) } & 3 & 1 & 7 & 2 & 6 & 4 & 5 \\ \text { G) } & 2 & 4 & 7 & 5 & 3 & 1 & 6\end{array}$

(les chiffres représentent les nombres des sept durées) développées par ellesmêmes en sept constellations. 
Alors que $A$ et $B$ sont formées à partir de chaque nombre dans l'ordre /ce qui donne une répétition horizontale-verticalel, les cinq autres groupes sont développés par eux-mêmes en une suite cyclique de permutation (11).

Exemple du développement de $C$ par lui-même (4-7-1-6-2-5-3) :

Groupe C

$\begin{array}{rrrrrrr}216 & 64 & 729 & 96 & 486 & 144 & 324 \\ 96 & 324 & 216 & 144 & 64 & 486 & 729 \\ 144 & 729 & 96 & 486 & 324 & 64 & 216 \\ 486 & 216 & 144 & 64 & 729 & 324 & 96 \\ 64 & 96 & 486 & 324 & 216 & 729 & 144 \\ 324 & 144 & 64 & 729 & 96 & 216 & 486 \\ 729 & 486 & 324 & 216 & 144 & 96 & 64\end{array}$

Les sept groupes ainsi obtenus sont superposés. Ce qui donne un contrepoint de quarante-neuf parties. Si les durées de chacune des parties sont d'une longueur extrême, par contre les durées situées entre chaque événement se perçoivent à une échelle beaucoup plus réduite. Elles donnent naissance à un rythme d'une grande variété, résultant de tous les groupes * aplatis ». Pour augmenter cette variété et éviter toute symétrie, les points de départ de chaque groupe sont décalés les uns par rapport aux autres suivant les durées centrales aplaties du groupe $A$ (symétrique de $B$ ), injectées en quelque sorte au début. (Les points de départ de chacun des groupes se font donc à doubles croches: $0,7,137,45,34$, et 13). Le résultat (linéaire aplati) de tous les groupes donne une véritable danse de durée entre les événements. Pour rendre plus sensibles ces constellations, elles furent rédigées et travaillées sous forme de graphique.

Quant aux sons, ils suivent une structure identique à partir de cette série d'origine :

Exemple 1

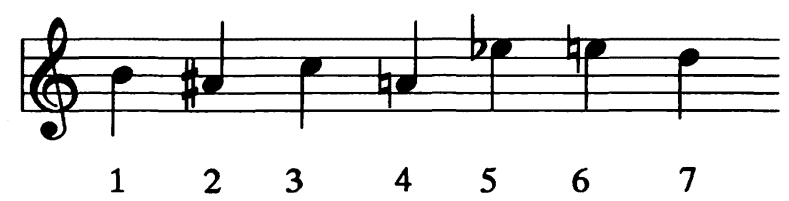

L'ordre de transposition est donné par les formes-mères alors que l'ordre des sons, une fois transposés, suit les permutations issues du développement des formes-mères par elles-mêmes, comme pour les durées.

Exemple avec le même groupe $C$ appliqué aux sons :
(1) Ainsi dans le groupe $C$, on a pris le quatrième nombre, le septième, le premier, etc., puis l'opération a été réitérée avec la nouvelle série obtenue, et ainsi de suite, jusqu'à ce que le cercle se soit refermé. la septième série rétablit toujours la série d'origine. 
Exemple 2

Densites. selon
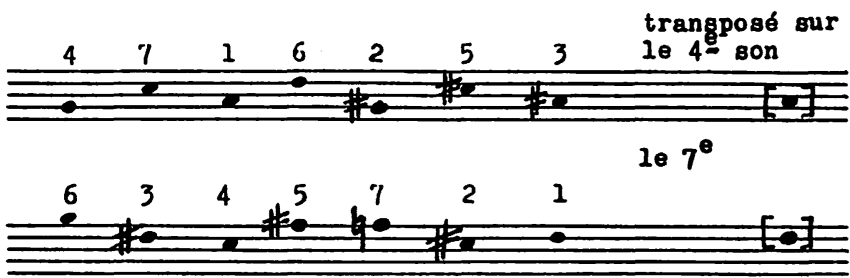

Ie $1^{\text {er }}$

d 5

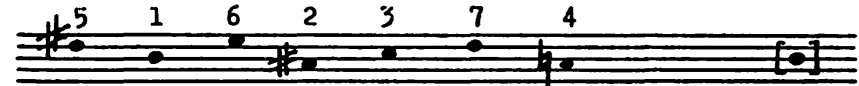

d. 4

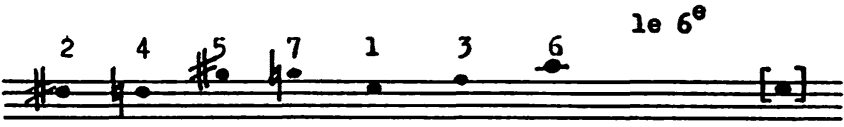

$102^{6}$

2 3

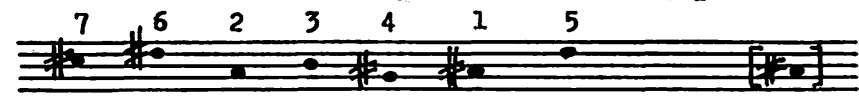

$105^{\circ}$

a 2

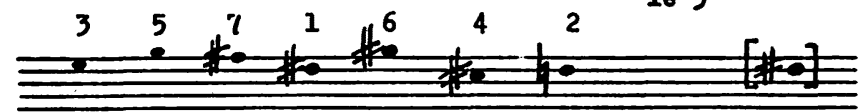

$103^{\theta}$

a 1

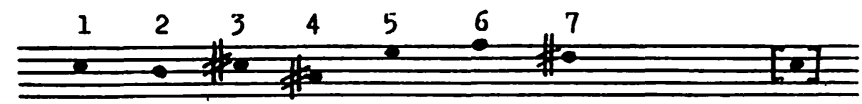

$$
\text { Groupe C (sons). }
$$

Par contre un unique mode d'intensité est appliqué à tous les groupes, empreinte issue des sept formes-mères :

$\begin{array}{lllllll}f f & f f & f & m f & p & p p & p p p \\ p p p & p p & p & m f & f & f f & f f f \\ m f & p p p & f f & p p & f f & p & f \\ p & f & p p & f f & p p p & f f & m f \\ p p & f f & p p p & p & f & m f & f f \\ f & f f f & p p p & f f & p p & m f & p \\ f f & m f & p p p & p & f & f f & p p\end{array}$

Pour concrétiser tout cela, un choix de sept formations d'instruments correspondra aux sept groupes-constellations de durées. Disposées stéréophoniquement pour aérer la perception, elles réaliseront sept musiques différentes. Précisons: en plus des timbres, de véritables «motifs obligés » 
qualifieront les groupes. Ces motifs obligés seront à la fois type d'entretien (référence au vocabulaire de Pierre Schaeffer) et manière d'être du son, extension de l'idée d'ornements, de \& manières », comme les nommaient euxmêmes les clavecinistes. Enfin, le nombre de sons participant aux harmonies (nommé « densité » dans l'exemple ci-dessus) dans certains cas, ou bien le registre, dans d'autres (piano, percussion), servira à identifier la position plus ou moins éloignée des formes développées par rapport aux formes-mères. Précisons encore: les très longues durées désignent l'intervalle entre deux moments et non pas des tenues de plusieurs minutes acoustiquement sursaturantes et physiquement impossibles pour certains instruments. Chacune des durées est donc représentée par une durée plus courte pour les groupes où cela s'imposait $(21$ doubles croches: $13,8,5,3,2,1)$. Par exemple, pour la durée 729 d.c., on aura 21 d.c. en son et le reste en silence 1708 d.c.), et ainsi de suite.

Illustrons maintenant nos timbres et motifs-obligés:

- groupe A, peaux-métaux. Ponctuel plus résonance, donc profil de chute double (rapide pour les peaux, lent pour les métaux) :

- groupe $B$, « ondes Martenot ». Un son fluctuant comme fréquence ou comme timbre, la vitesse du mouvement pouvant être altérée par l'environnement sonore ;

- groupe $C$, l'ensemble lames-lattes-tubes: un neume-anacrouse rapide précède un point de chute (accent) à distance variable. Ce sont des neumes ${ }^{(2)}$ d'avertissement (cf. exemple 3) ;

- groupe $D$, les cordes, style pluie mélodique de petites notes lleur nombre dépendra de la durée représentée) ;

- groupe E, le piano en clusters (bande de sons) distribués sur sept registres;

- groupe F, percussions ligneuses (blocs chinois, guiro, maracas, claves, etc.) en batteries rapides, style pic-bois ou en raclement, style insectes ;

- groupe $G$, les vents, disposés de part et d'autre du public, exécutent des neumes d'espace (le son se déplaçant d'un lieu à un autre).
(2) Nom donné aux signes mélodiques dans le plain-chant. 
Exemple 3

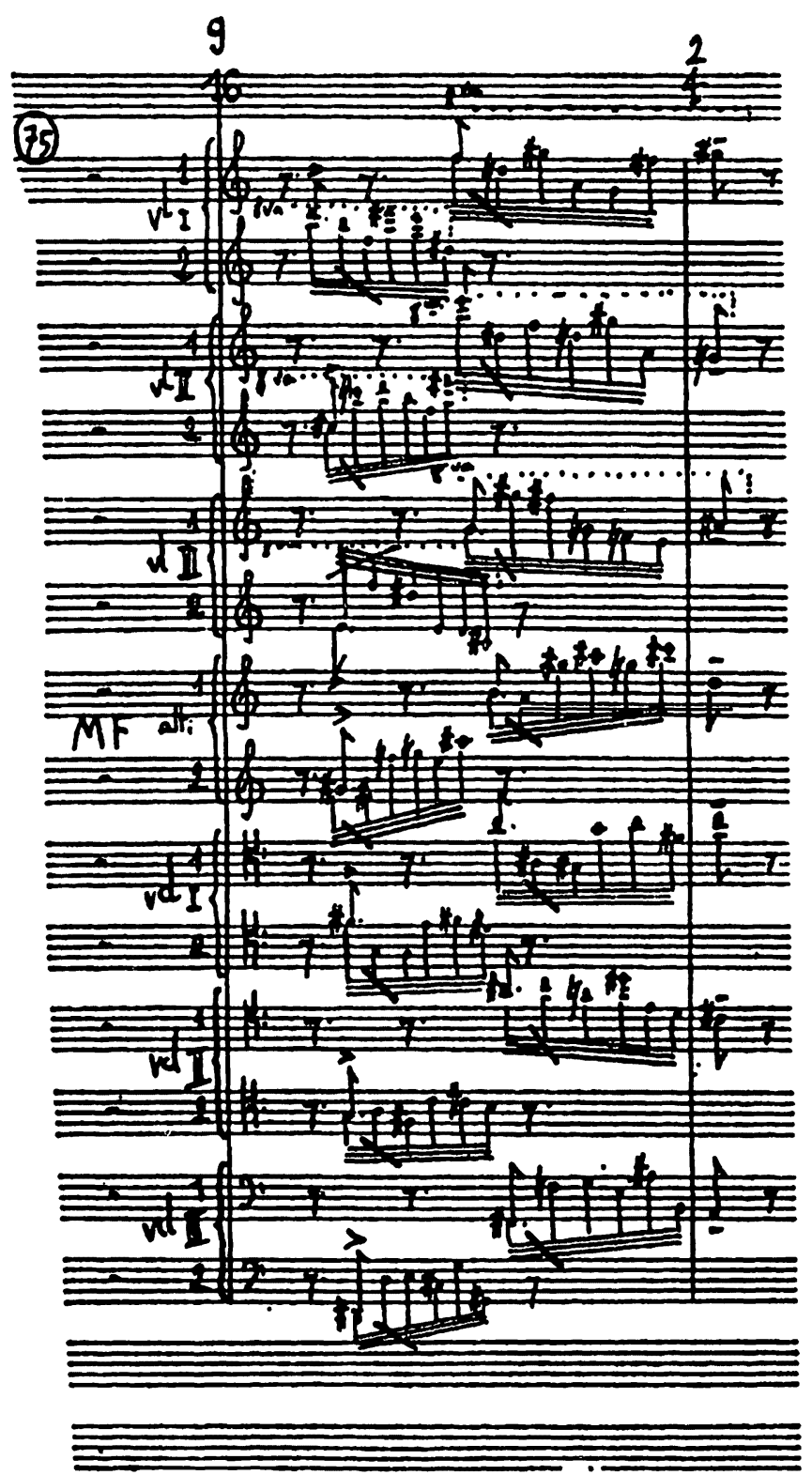


Au sujet du Martenot, de l'ensemble lames-lattes-tubes et des vents, une même série limitée de sept neumes est utilisée dans les trois cas, ce qui donne des à des échelles et dans des musiques différentes: Torculus resopinus (. . . .) porrectus flexus $(\cdot \ldots)$, torculus $(. \cdot \cdot)$, porrectus $(\cdot$.$) , podatus (. \cdot)$, clivis $(\cdot)$, punctum (.). Ainsi, un torculus, traduit dans ces trois groupes, pourra donner:

aux ondes Martenot:

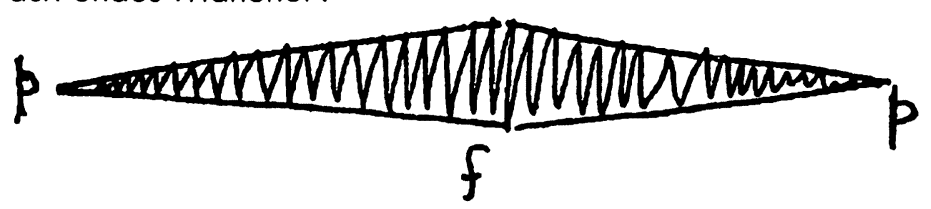

au xylophone (anacrouse rapide) :

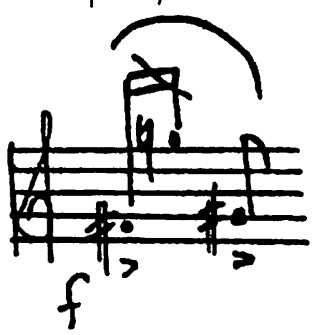

aux vents (en prenant comme convention : inférieur = gauche et supérieur $=$ droite) ce mouvement spatial :

Exemple 4

\section{Groupe de droite}

Groupe de gauche

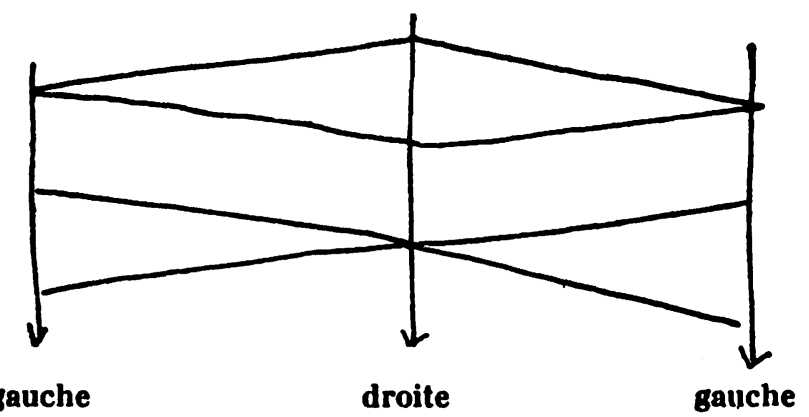

Une fois toute cette organisation soigneusement établie, prévue, calculée, il ne restait donc plus qu'à réaliser. Enfin le rêve étoilé pouvait prendre forme, les novæ se déchaîner ${ }^{(3)}$. C'est alors que l'imprévisible ${ }^{(4)}$ se produisit. L'imagination-organisation fut confrontée avec l'imagination-composition, impérieuse, affamée d'immédiat et farouchement « en-temps » /cf. la distinction de Xenakis avec l'« hors-temps »/ qui se trouvait acculée à une passivité révoltante : à ce
(3) Signalons ici que l'œuvre est dédiée à Olivier Messiaen.

(4) Citons une toute petite phrase de Pierre Boulez que presque personne ne remarque, bien dissimulée qu'elle est dans son article « Éventuellement $:$ : $\|$ n'y a de création que dans l'imprévisible devenant né cessité * (La Revue musicale, $n^{\circ} 212$, avril 1952, p. 141 ; repris in Relevés d'apprenti.) 
point, l'œuvre, à la rigueur, aurait pu être confiée à une machine réalisatrice ! Je me trouvai donc dans l'obligation d'interrompre ma rédaction, paralysé par ce dilemme, n'ayant même plus le choix d'annihiler le travail déjà fait - il était déjà trop vivant et hantant de possibilités en soi. D'autre part, réaliser bêtement l'organisation prévue revenait à une démission de compositeur.

Rapprocher les deux imaginations (organisation et composition).

Attendre. (Qu'agissent les hauts-fourneaux de l'imagination sur ces monolithes séduisants mais gelés.)

Être à l'écoute.

Peu à peu, à travers les attaques de l'imagination II, une solution se dégage: la fixité première de l'organisation sera progressivement détruite par vagues successives (elles-mêmes organisées) au profit d'échanges et de multiplications décidés sur-le-champ selon les situations immédiates.

Par exemple, le groupe lattes-lames-tubes pourra imiter les cordes et leurs petites notes;

Exemple 5

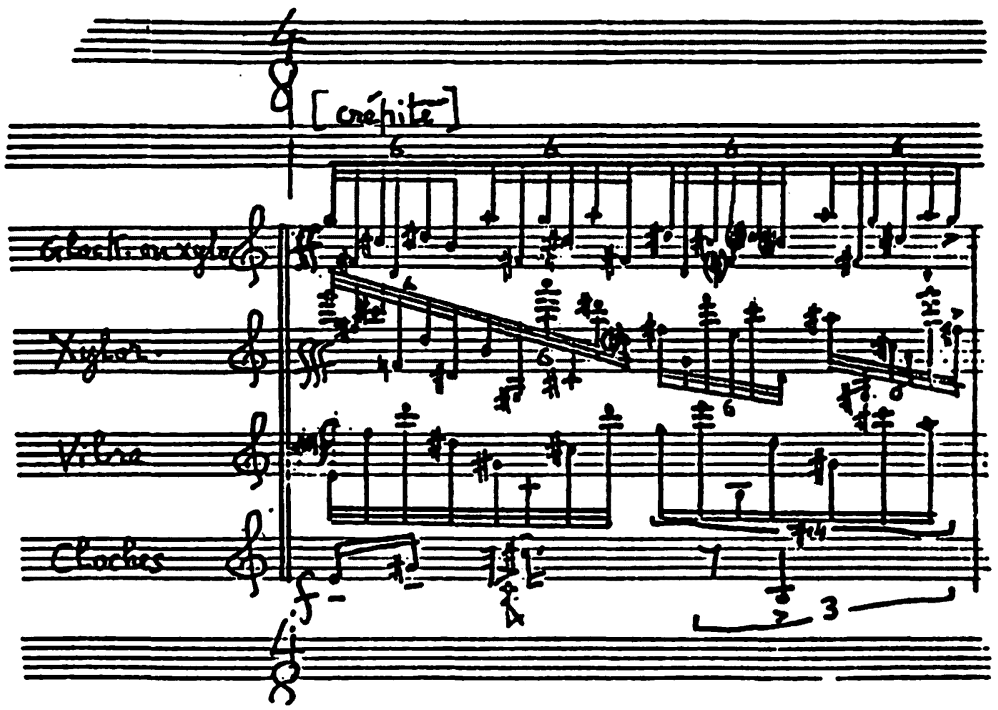

les neumes d'espace des vents se transformeront en neumes de timbre aux cordes, etc. L'action de ces échanges étant multilatérale, de véritables contagions sont parfois créées où l'un ou l'autre des modes d'être du son prendra le dessus et où, à d'autres moments, des formes hybrides naîtront de la fusion de 
deux ou plusieurs éléments. (Exemple : $B$ (fluctuation des ondes) + E (clusters) donnerait en $D$ (groupe des cordes) un cluster-glissando. A (ponctuel + résonance) + D (mélodies en petites notes) en $G$ (les vents), un sforzando bref à gauche suivi d'une désinence mélodique diminuendo en guise de résonance à chute longue.) Cependant, les événements, bien qu'ils soient transformés et que d'autres s'y agglutinent par attraction, arrivent toujours à l'endroit prévu dans le temps. Comme dans un feu de bois, la matière change en se consumant, mais les traces laissées gardent l'empreinte de la forme du bûcher initial. Dans ce jeu d'échanges et de contagion transformatrice, la progression générale est aimantée vers une liberté de plus en plus grande.

C'est pourquoi le morceau se termine avec l'apparition de la voix qui n'est justifiée par aucune raison d' "organisation », et elle témoigne en sa gratuité même de cette liberté conquise. Nul effet de théâtre, plutôt une ambiguité de timbre où un mi venu des ondes s'écarte aux vents pendant une véritable explosion d'orchestre d'où il émerge à la voix solo au fond de la scène. Est-ce une voix ? Est-ce un instrument? L'auditeur ne peut identifier ce qui s'est passé, mais seulement avoir le sentiment d'une sorte de naissance.

Conclure ? Oui, par le paradoxe d'une destruction organiquement constructrice (méditons Lao-Tzeu...), et surtout dans la musique en fêtant cette liberté.

BOULEZ, Pierre (1966), Relevés d'apprenti, Paris, Sevil. 